\title{
A Survey of Practice Patterns for Rehabilitation Post Elbow Fracture
}

\author{
Joy C. MacDermid", ${ }^{*}$, Joshua I. Vincent ${ }^{2}$, Leah Kieffer ${ }^{3}$, Ashley Kieffer ${ }^{3}$, Jennifer Demaiter ${ }^{3}$ and \\ Stephanie MacIntosh ${ }^{3}$
}

\author{
${ }^{I}$ Rehabilitation Science, McMaster University, School of Rehabilitation Science, Hamilton, Ontario, Canada and Hand \\ and Upper Limb Center, St. Joseph's Health Center, London, Ontario, Canada \\ ${ }^{2}$ University of Western Ontario, Health and Rehabilitation Sciences, London, Ontario, Canada \\ ${ }^{3}$ University of Western Ontario, School of Physical Therapy, London, Ontario, Canada
}

\begin{abstract}
Background and Purpose: Elbow fractures amount to $4.3 \%$ of all the fractures. The elbow is prone to stiffness after injury and fractures can often lead to significant functional impairment. Rehabilitation is commonly used to restore range of motion (ROM) and function. Practice patterns in elbow fracture rehabilitation have not been defined. The purpose of this study was to describe current elbow fracture rehabilitation practices; and compare those to the existing evidence base.

Methods: Hand therapists $(\mathrm{n}=315)$ from the USA $(92 \%)$ and Canada $(8 \%)$ completed a web-based survey on their practice patterns and beliefs related to the acute (0-6 weeks) and functional (6-12 weeks) phases of elbow fracture rehabilitation.

Results: More than $99 \%$ of respondents agreed that fracture severity, co-morbidities, time since fracture, compliance with an exercise program, psychological factors, and occupational demands are important prognostic indicators for optimal function. Strong agreement was found with the use of patient education (95\%) and active ROM (86\%) in the acute stage while, home exercise programs (99\%), active ROM (99\%), stretching (97\%), strengthening (97\%), functional activities (ADLs and routine tasks) (97\%), passive ROM (95\%), and active assisted ROM (95\%) were generally used in the functional stage. The most commonly used impairment measures were goniometry (99\%), Jamar dynamometry $(97 \%)$, and hand held dynamometry (97\%). Agreement on the use of patient-reported outcome measures was very minimal $(1.3 \%-35.6 \%)$.

Conclusions: Exercise, education, and functional activity have high consensus as components of elbow fracture rehabilitation. Future research should focus on defining the optimal dosage and type of exercise/activity, and establish core measures to monitor outcomes of these interventions.
\end{abstract}

Keywords: Elbow fracture, hand therapist, intervention, outcome measures, prognostic factors, rehabilitation, survey.

\section{INTRODUCTION}

Elbow joint fractures make up $4.3 \%$ of all fractures [1]. The elbow is prone to stiffness after injury and fractures can often lead to substantial functional impairment [1]. Early rehabilitation can make the difference between a functional limb and an extremity with permanent functional loss [2]. The goal of treatment is to restore motion and function. Fractures typically result in a loss of elbow extension range of motion [3]. Although many narrative reviews suggest that therapy should be routinely used following elbow fracture, a recent review of the evidence on optimal rehabilitation of post-traumatic elbow stiffness noted the paucity of evidence, a lack of clarity in defining rehabilitation interventions, and a reliance on expert opinions with respect to this topic [4]. This state of the evidence provides challenges to therapists wishing to utilize an evidence-based practice approach.

*Address correspondence to this author at the Hand and Upper Limb Centre, St. Joseph's Health Centre, 268 Grosvenor Street, London, Ontario, N6A 4L6, Canada; Tel: 519-646-6100, Ext. 64636; Fax: 519-646-6049;

E-mail: macderj@mcmaster.ca
Understanding the practices of experienced therapists in rehabilitation following elbow fracture could define areas of expert consensus and gaps between practice and current evidence [5].

Elbow fracture rehabilitation can begin within days of the initial injury, and continue throughout the bone healing and bone remodeling phases. The objectives of rehabilitation, the appropriateness of specific interventions, and the relative effectiveness of the treatment will vary depending upon which stage of rehabilitation is being addressed. Therefore, we divided our line of questioning into two key time periods during fracture recovery; the acute bone healing stage and the functional rehabilitation stage. The acute bone healing stage was defined as a stage of non-union, usually between 0-6 weeks post injury or until union occurs. The functional rehabilitation stage was defined as a stage where bone healing is confirmed by radiographs, generally 6-12 weeks post-injury and onward.

The objective of this study was to describe the practice patterns of hand therapists with respect to assessment, 
treatment, prognosis and outcome evaluation in elbow fracture rehabilitation.

\section{MATERIALS AND METHODS}

\section{Survey Development}

The survey was designed to acquire information on assessment procedures, prognostic indicators, interventions, and outcome measures. Review of the literature, a previous clinical practice survey, and discussion with clinicians were used to identify categories and responses to the pilot survey. Multiple drafts and edits were performed by the investigating team to refine the survey and response options. Following this a written version of the survey was piloted using a convenience sample of 10 physical therapists, who provided feedback on the format and acceptability of the content. Feedback resulted in slight modifications to the items to improve the clarity of the questions and shortening of the length of the survey. The finalized version of the survey was mounted using Mr. Interview ${ }^{1}$, a software program for webbased survey administration.

The final survey consisted of 165 questions classified into 6 broad sections with sub-sections under each category. The first category of the survey comprised of 31 questions under 2 sub-sections regarding the specific examination procedures including radiological and other diagnostic tests that were used to determine management of elbow fractures. The second category with 14 questions inquired which prognostic indicators were thought to be important in determining the optimal functional outcome of patients with elbow fractures. The third category was split into the acute bone healing stage (37 questions under 6 sub-sections) and the functional rehabilitation stage (48 questions under 6 subsections) and the questions were directed to_identify which interventions were used most often and their perceived effectiveness. The fourth section comprised of a list of 19 outcome measures both impairment and self-reported measures and the respondents were asked to identify the ones they used. The final two sections included questions pertaining to the demographic characteristics of the respondents as well as a description of their clinical context and caseload.

Structured response options were provided for each question using a checkbox method and in most of the subsections of the survey there was an "other" option that could be clicked and respondents were able to type in the responses that were not included in the survey.

\section{Population Sampling and Data Collection}

This study was approved by the University of Western Ontario Ethics Review Board. Informed consent was obtained from all respondents in the survey. Following the ethics board approval the survey was distributed by broadcast emails to The American Society of Hand Therapists (ASHT), The Canadian Physiotherapy Association (CPA), and placed a web link within a newsletter of the Orthopedic Division of the American Physical Therapy Association (APTA). It was first sent out to members of ASHT during the month of April 2008 with a second request to follow a few weeks later. Next, the survey

${ }^{1}$ Mr. Interview, Dimensions ${ }^{\mathrm{TM}}$, IBM SPSS was sent to both CPA and APTA during the months of May and June 2008, respectively, also with a second request to follow. A third last request was also sent out to all three associations. The final data was collected 12 weeks after the initial broadcast email. A flow chart of the methodological process is shown in Fig. (1).

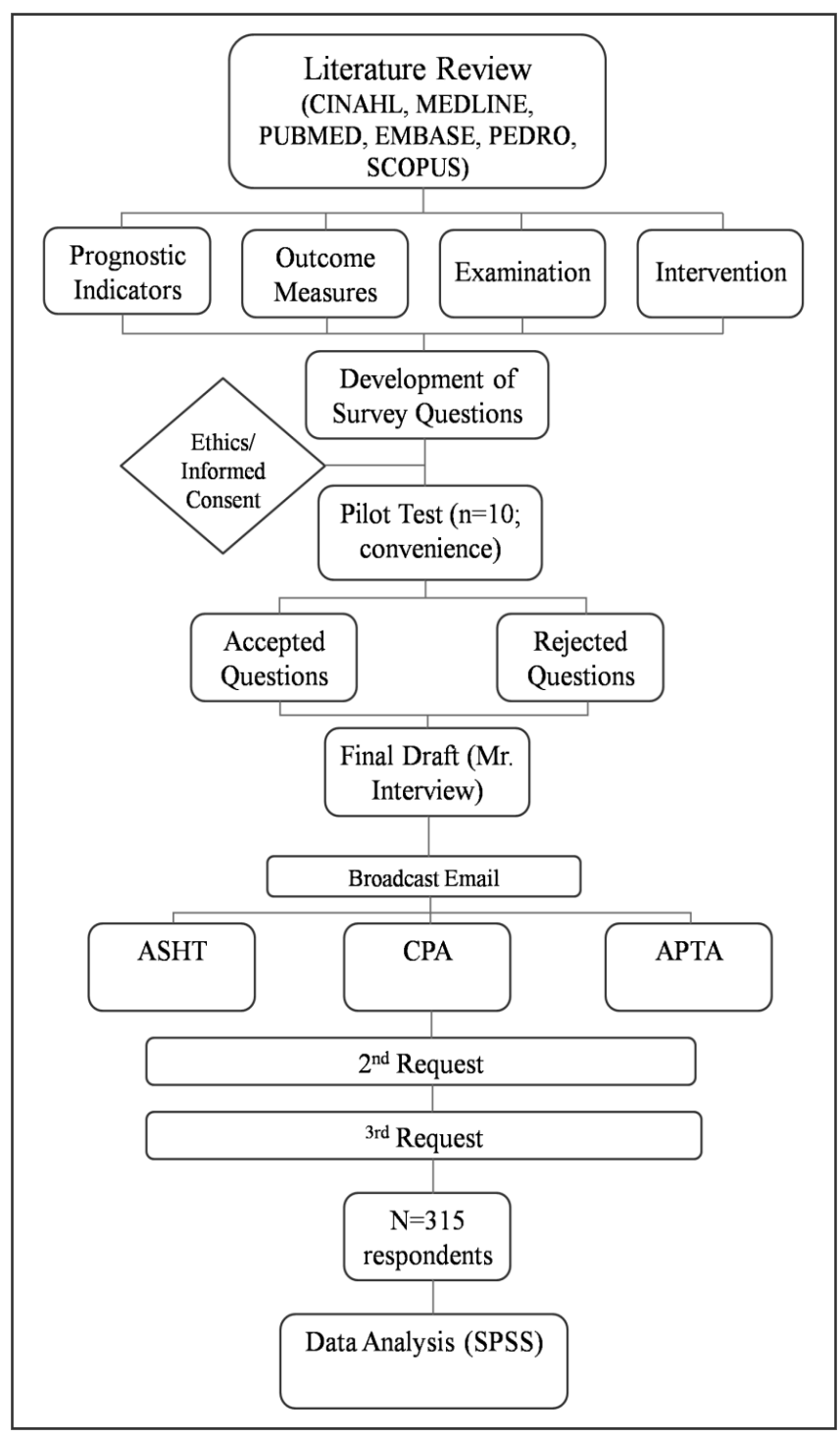

Fig. (1). Flow chart depicting the study methods.

\section{Data Analysis}

After respondents completed the survey the data was collected and directly transferred from Mr. Interview into the Statistical Package for the Social Sciences (SPSS) version 15.0. Descriptive statistics were run to get details pertaining to examination procedures, prognostic indicators, interventions, outcome measures, demographic characteristics, and caseload information. Data were reported as percentages and frequency distributions where appropriate.

\section{RESULTS}

\section{Subjects}

The survey targeted hand therapists $(n=315)$ throughout the United States $(n=290 ; 92 \%)$ and Canada $(n=25 ; 8 \%)$. 
$89 \%$ of the respondents were certified hand therapist (CHT). From the demographic characteristic section of the survey, it was found that the majority of the respondents were female $(89 \%)$ in the age range of $40-49$ years old (See Table 1). The majority of respondents $(71 \%)$ reported practicing greater than 15 years. The majority $(80 \%)$ of the respondents were providing direct patient care in urban practices. The highest degree attained by $61 \%$ of respondents was a baccalaureate degree; of which $84 \%$ were occupational therapists and $6 \%$ were physiotherapists. Lastly, there was a wide variety of practice settings with the most common facilities being acute care hospitals with outpatient clinical services (30\%), therapist owned outpatient therapy clinics (27\%), and physician owned outpatient therapy clinics (23\%) (See Table 1).

\section{Examination Procedures}

Acute Stage: Radiographs were the most commonly utilized imaging technique, with $66 \%$ of the respondents reporting that they "always or frequently" used them. Other imaging techniques were infrequently used including: angiography $(1 \%)$, bone scan $(4 \%)$, computed tomography (CT) scan (11\%), and magnetic resonance imaging (MRI) $(15 \%)$. Greater than $90 \%$ of the respondents answered that they "always or frequently" used the following assessment methods: observation for deformity $(96 \%)$, assessment of the unaffected surrounding joints (95\%), and palpation of the area for swelling $(90 \%)$. Least agreement was found for the use of vibration/tap test (4.8\%) (See Table 2).

Rehabilitation Stage: During the functional rehabilitation stage, radiographs were reported as "always or frequently" being consulted by the majority (58\%) of the respondents. Again imaging techniques like angiography $(1.6 \%)$, bone scans $(3.2 \%)$, and CT scans $(10.1 \%)$ were rarely used. A wide variety of assessment techniques were reported as commonly used by therapists during the rehabilitation stage. The greatest endorsement $(>80 \%$ of respondents reporting "always or frequently"using) was for the assessment of: ROM (99\%), joint deformities (94\%), functional performance $(94 \%)$, unaffected surrounding joints $(93 \%)$, grip strength $(89 \%)$, elbow (Biceps/triceps) strength $(86 \%)$, swelling $(86 \%)$, and pain (81\%). Similar to the acute stage the least used assessment technique was the vibration/tap test (10.7\%) (See Table 3).

\section{Prognostic Indicators}

Greater than $99 \%$ of respondents reported the following factors to be having very high prognostic relevance: compliance with an exercise program (96\%), classification/severity of fracture (93\%), time since fracture (91\%) and co-morbidities $(80 \%)$. The least consensus was obtained on the level of education (18\%) and ergonomic changes at the worksite $(30 \%)$ as prognostic factors. Moderate consensus was obtained for all the other prognostic factors (See Table 4). When respondents were asked to provide any other prognostic indicators, some listed additional factors including: pain tolerance, psychological factors, social factors, socioeconomic factors, and accessibility.
Table 1. Demographic Characteristics

\begin{tabular}{|c|c|}
\hline Demographic and Caseload Information & $\begin{array}{c}\text { Percentage of } \\
\text { Respondents (\%) }\end{array}$ \\
\hline \multicolumn{2}{|l|}{ Gender } \\
\hline Female & 88.8 \\
\hline Male & 11.1 \\
\hline \multicolumn{2}{|l|}{ Age Group (Years) } \\
\hline $20-29$ & 1.0 \\
\hline $30-39$ & 22.2 \\
\hline $40-49$ & 40.5 \\
\hline $50+$ & 36.3 \\
\hline \multicolumn{2}{|l|}{ Profession } \\
\hline Certified Hand Therapist (CHT) & 89 \\
\hline OT & 78.9 \\
\hline PT & 11 \\
\hline \multicolumn{2}{|l|}{ Country of practice } \\
\hline USA & 92 \\
\hline Canada & 8 \\
\hline \multicolumn{2}{|l|}{ Years in Practice } \\
\hline$<5$ & 1.0 \\
\hline $5-10$ & 11.1 \\
\hline $11-15$ & 16.6 \\
\hline$>15$ & 71.2 \\
\hline \multicolumn{2}{|l|}{ Highest Degree Attained } \\
\hline Diploma & 1.3 \\
\hline Baccalaureate & 60.6 \\
\hline Entry-level masters & 15.7 \\
\hline Advanced masters & 19.2 \\
\hline Clinical doctorate & 2.2 \\
\hline $\mathrm{PhD}$ & 1.0 \\
\hline \multicolumn{2}{|l|}{$\%$ of Direct Patient Care } \\
\hline $0-25 \%$ & 2.2 \\
\hline $26-50 \%$ & 1.6 \\
\hline $51-75 \%$ & 7.7 \\
\hline $76-100 \%$ & 88.5 \\
\hline \multicolumn{2}{|l|}{ Location of Facility } \\
\hline Rural & 19.7 \\
\hline Urban & 80.3 \\
\hline
\end{tabular}

\section{Treatment}

Acute Stage: During the acute bone healing stage education regarding precautions, rest and activity 
Table 2. Use of Examination Procedures During Acute Bone Healing Phase of Elbow Fractures

\begin{tabular}{|c|c|c|}
\hline S. No. & Examination Procedure & \% of Respondents who Reported as Frequently/Always Using \\
\hline \multicolumn{3}{|c|}{ Imaging Techniques } \\
\hline 2 & MRI & 14.5 \\
\hline 3 & CT scan & 10.9 \\
\hline 5 & Angiography & 1.1 \\
\hline \multicolumn{3}{|c|}{ Manual Assessment Techniques } \\
\hline 1 & Observation for deformity & 95.6 \\
\hline 2 & Assessments of unaffected surrounding joints & 94.6 \\
\hline 6 & Vascular assessment & 52.4 \\
\hline 7 & Palpation of fracture site for union & 34.5 \\
\hline 8 & Vibration/tap test & 4.8 \\
\hline
\end{tabular}

Table 3. Use of Examination Procedures at Rehabilitation Phase of Elbow Fracture

\begin{tabular}{|c|c|c|}
\hline S. No. & Examination Procedure & \% of Respondents who Reported as Frequently/Always Using \\
\hline \multicolumn{3}{|c|}{ Imaging Techniques } \\
\hline 2 & MRI & 15.8 \\
\hline 3 & CT scan & 10.1 \\
\hline 5 & Angiography & 1.6 \\
\hline \multicolumn{3}{|c|}{ Manual Assessment Techniques } \\
\hline 1 & Range of motion & 99.1 \\
\hline 2 & Observation for deformity & 94 \\
\hline 6 & Elbow muscle strength & 86.1 \\
\hline 7 & Swelling & 85.7 \\
\hline 8 & Pain & 80.7 \\
\hline 9 & Sensory evaluation & 67.7 \\
\hline 10 & Vascular assessment & 45.7 \\
\hline 11 & Palpation of fracture site for union & 42.6 \\
\hline 12 & Vibration/tap test & 10.7 \\
\hline
\end{tabular}

modifications, and pain management were "always or frequently" a component of treatment post elbow fracture. AROM and active assisted ROM (AAROM) were "always or frequently" used by $87 \%$ and $75 \%$ of clinicians respectively. Passive ROM (PROM) (32\%) and stretching $(26 \%)$ were included less frequently, and strengthening was "always or frequently"incorporated by very few (4\%). Posture (72\%) and ergonomic modification (40\%) 
Table 4. Prognostic Indicators that were Thought to be Somewhat Important/Very Important in Elbow Fracture Rehabilitation

\begin{tabular}{|c|c|c|c|}
\hline S. No. & Prognostic Indicator & Very Important (a) in \% & Somewhat Important (b) in \% \\
\hline 1 & Compliance of exercise program & 96.2 & 3.2 \\
\hline 3 & Time since fracture & 90.9 & 8.8 \\
\hline 4 & Co- morbidities & 80.3 & 19.4 \\
\hline 6 & Previous history of elbow fracture & 68.4 & 29.1 \\
\hline 7 & Age & 60.6 & 37.5 \\
\hline 8 & Occupational demands & 60.3 & 38.8 \\
\hline 9 & Worker's physical fitness & 44.2 & 53.9 \\
\hline 13 & Ergonomic changes at workplace & 30.6 & 65.0 \\
\hline 14 & Level of education & 18.0 & 63.6 \\
\hline 15 & Others* & 28.9 & 43.5 \\
\hline
\end{tabular}

*Other factors included pain tolerance, psychological factors, social factors, socioeconomic factors, accessibility etc.

interventions were included to a lesser extent. Orthotic devices were commonly, but not consistently, used by clinicians. Static splinting (58\%) was most commonly used, whereas all other orthotic devices were reported as being used "always or frequently" by less than $15 \%$ of therapists (See Table 5).

Therapeutic modalities were not frequently used in treatment during the acute stage. The combination of rest, ice, compression, and elevation (RICE) (77\%) was most frequently used, followed by heat (wax therapy hydrocollator packs) (63\%), compression alone (61\%), and ice alone (50\%) (See Table 5). All other modalities were "always or frequently" used by less than $20 \%$ of those surveyed. Common treatment areas reported in addition to the elbow were the wrist (81\%) and shoulder (74\%), with a small percentage $(15 \%)$ "always or frequently" treating the neck. The strongest consensus about modalities that were never used included magnetic field therapy (97\%) acupuncture (97\%), pneumatic pump (87\%), and laser $(86 \%)$. CPM and taping were never used by $68 \%$ and $64 \%$ of therapists, respectively. The respondents were given an option to provide some other treatment techniques they use other than what was listed in the survey. These responses included myofascial release techniques, multi-angle isometrics, patient education on the healing process, high voltage pulsed galvanic current, and progressive static splinting.

In terms of effectiveness of the treatments, both AROM and AAROM were reported to be very effective therapeutic modalities by $83 \%$ of respondents, followed by RICE (74\%), compression (71\%), heat (67\%) and ice $(57 \%)$. All other therapeutic modalities were reported to be very effective by

\section{Table 5. Treatment Techniques Commonly Used During the Acute Phase of Elbow Fractures}

\begin{tabular}{|c|l|c|}
\hline S. No. & \multicolumn{1}{|c|}{ Interventions } & \% of Respondents who Reported Frequently/Always Using \\
\hline \hline 1 & Patient education on precautions & 94.8 \\
\hline 2 & Patient education on rest and activity modification & 87.9 \\
\hline 3 & Active range of motion exercises & 86.6 \\
\hline 4 & Patient education on pain management & 86.2 \\
\hline 5 & RICE - rest, ice, compression, elevation & 76.8 \\
\hline 6 & Active-assisted range of motion exercises & 75 \\
\hline 7 & Posture education & 72.4 \\
\hline 8 & Heat modalities & 63 \\
\hline 9 & Compression wrap/tensor & 60.5 \\
\hline 10 & Static splinting & 58 \\
\hline
\end{tabular}




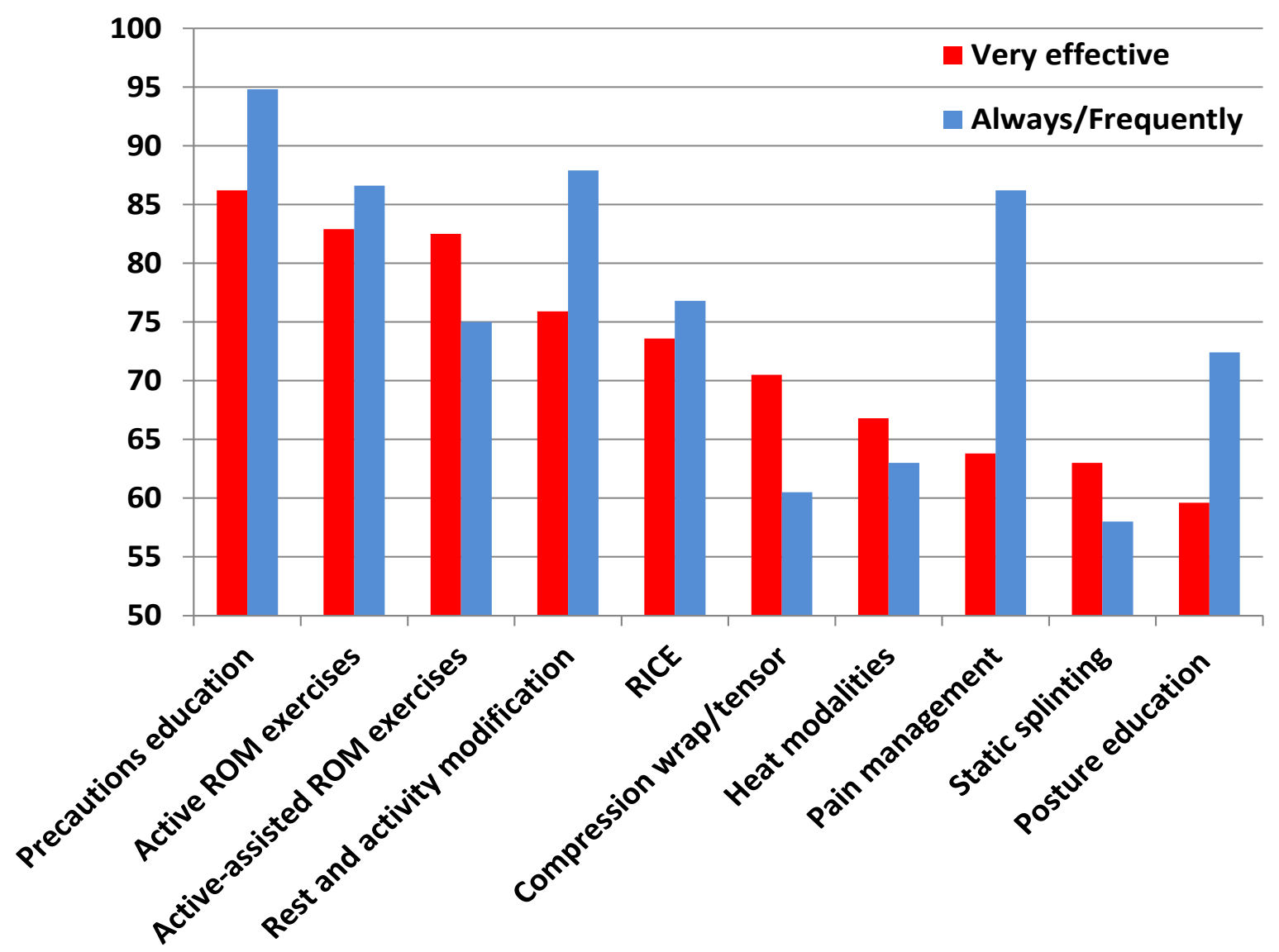

Fig. (2). Treatment techniques commonly used and their perceived effectiveness during the acute phase of elbow fractures.

not more than $40 \%$. Education regarding precautions $(86 \%)$, and treating the wrist $(91 \%)$ and shoulder (85\%) was reported as very effective interventions by most therapists. A bar graph of the common treatments used in the acute stage and their perceived effectiveness is shown in Fig. (2).
Rehabilitation Stage Goals for therapy during the functional rehabilitation stage differ from those of the acute stage. During the rehabilitation stage, therapists focus more on the restoration of function and less on pain and edema management. With respect to treatment involving exercise prescription, surveyed therapists most commonly agreed on prescribing elbow AROM (99\%), elbow stretches (98\%),

Table 6. Treatment Techniques Commonly Used During the Functional Rehabilitation Phase of Elbow Fractures

\begin{tabular}{|c|l|c|}
\hline S. No. & \multicolumn{1}{|c|}{ Interventions } & \% of Respondents who Reported Frequently/Always Using \\
\hline \hline 1 & Active range of motion exercises & 99.4 \\
\hline 2 & Home program & 99.4 \\
\hline 3 & Stretching exercises & 97.5 \\
\hline 4 & Strengthening exercises & 97.2 \\
\hline 5 & Functional activity training & 96.5 \\
\hline 6 & Passive range of motion exercises & 95.2 \\
\hline 7 & Active-assisted range of motion exercises & 94.7 \\
\hline 8 & Education on precautions & 93.4 \\
\hline 9 & Heat modalities & 89 \\
\hline 10 & Rest and activity modification & 87.9 \\
\hline 11 & Patient education on pain management & 87.3 \\
\hline 12 & Massage & 80.4 \\
\hline 13 & Mobilization with movement & 69.4 \\
\hline
\end{tabular}




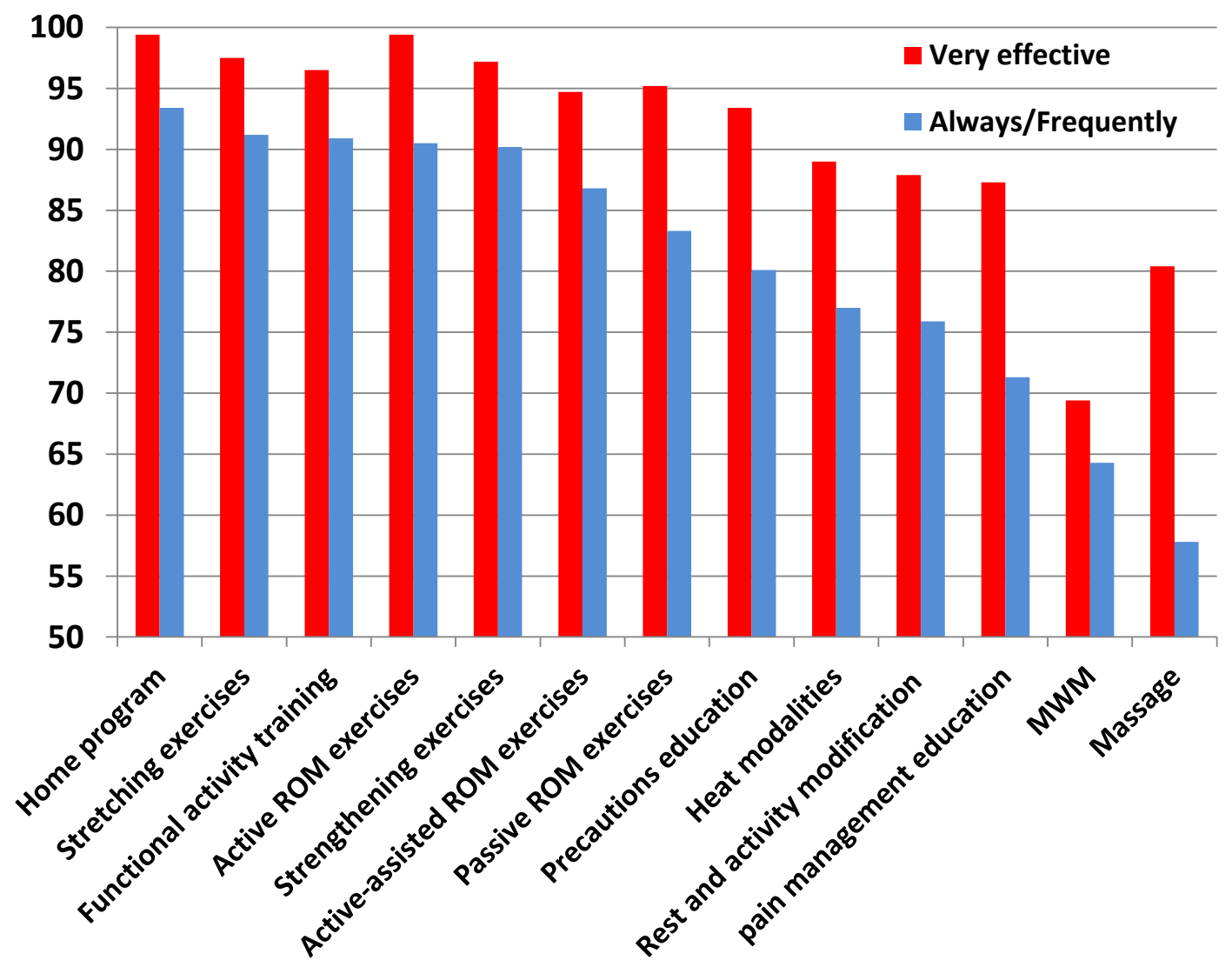

Fig. (3). Treatment techniques commonly used and their perceived effectiveness during the functional rehabilitation phase of elbow fractures.

strengthening exercises about the elbow (97\%), functional exercises (97\%), PROM (95\%) and AAROM (95\%).

Therapists also reported that patient education is an important part of rehabilitation post elbow fracture. The highest consensus was for teaching home program to the patient $(99 \%)$. The majority of therapists also responded that education about movement/range precautions (93\%), the balance between rest and activity with regards to healing $(88 \%)$, and techniques for pain management $(87 \%)$ were commonly used (See Table 6).

By far the most commonly used modality in the functional rehabilitation stage was heat $(89 \%)$. There was little to no use of acupuncture, magnetic field therapy, or ionization. Manual therapy techniques most commonly employed were massage, followed by mobilization with movement (MWM) and glides. Slightly more than half of the therapists reported the use of myofascial release, and oscillatory techniques. Only one quarter of the respondents used manipulation.

Orthotic devices were not used commonly in the rehabilitation stage when compared to its utilization in the acute stage. Less than half of the therapists reported prescribing orthotics for patients. Finally, the majority of therapists also treated the wrist and shoulder following an elbow fracture. The respondents were given an option to provide some other treatment techniques they use other than what was listed in the survey. Their responses included weight bearing exercises, joint protection, functional casting, and serial static splinting.
Implementing a home exercise program (93\%), stretching (91\%), AROM (91\%), strengthening (90\%), and functional activities training $(90 \%)$ were reported to be very effective at this stage by the majority of respondents. A slightly smaller number reported that heat $(77 \%)$ was a very effective modality. All other therapeutic modalities were reported to be very effective by less than $50 \%$ of Respondents. When asked about the perceived effectiveness of soft tissue and mobilization techniques, MWM was most often found to be very effective $(65 \%)$, followed by massage $(58 \%)$. Treating the wrist $(84 \%)$ and shoulder $(76 \%)$ were reported as very effective interventions by most therapists. Overall, perceived effectiveness was aligned with the frequency of use. A bar graph of the common treatments used in the rehabilitation stage and their perceived effectiveness is shown in Fig. (3).

\section{Outcome Measures}

The most common outcome measures that were reported as being used "always or frequently" were the impairment measures including: goniometry (99\%), Jamar dynamometry (97\%), hand held dynamometry (HHD) (97\%), and manual muscle testing (MMT) (89\%) (See Table 7). The numeric pain rating scale (NPRS) (84\%) was the only self-reported measure that was agreed upon by all respondents as "always or frequently" used. The least commonly endorsed outcomes measures (i.e. those that were reported as "never used") were mostly the self-reported outcomes; the Canadian Occupational Performance Measure (COPM) (93\%), short form 12 and 36 (92\%), and the Upper Extremity Functional 
Table 7. Use of Impairment Measures by Hand Therapists in Elbow Fracture Rehabilitation

\begin{tabular}{|c|l|c|}
\hline S. No. & \multicolumn{1}{|c|}{ Impairment Measure } & \% of Respondents who Reported as Frequently/Always Using \\
\hline \hline 1 & Goniometry & 98.4 \\
\hline 2 & Jamar grip strength & 97.2 \\
\hline 3 & Hand Held Dynamometer & 96.9 \\
\hline 4 & Manual muscle testing & 88.7 \\
\hline 5 & Arm circumference & 72.2 \\
\hline 6 & Semmes-Weinstein monofilaments & 38.2 \\
\hline 7 & Volumetrics & 21.1 \\
\hline 8 & 2-point discrimination & 17.3 \\
\hline 9 & Isokinetic dynamometry & 7.3 \\
\hline 10 & Minnesota rate of manipulation test & 6.4 \\
\hline 11 & Jebson's hand function test & 5.8 \\
\hline 12 & Others* & 13 \\
\hline
\end{tabular}

*Other impairment measures include 9 hole peg test, Valpar 4, Purdue pegboard, Pinch meter, O'Connor tweezer test etc.

Table 8. Use of Self-Report Measures by Hand Therapists in Elbow Fracture Rehabilitation

\begin{tabular}{|c|l|c|}
\hline S. No. & \multicolumn{1}{|c|}{ Patient Self-Reported Outcome Measure } & \% of Respondents who Reported as frequently/Always Using \\
\hline \hline 1 & Numeric pain rating scale (NPRS) & 83.6 \\
\hline 2 & Visual analogue scale (VAS) & 50.5 \\
\hline 3 & Disabilities of the arm hand and shoulder (DASH) & 35.6 \\
\hline 4 & Patient rated elbow evaluation (PREE) & 6.3 \\
\hline 5 & Patient specific functional scale (PSFS) & 5.1 \\
\hline 6 & Upper extremity functional scale (UEFS) & 1.7 \\
\hline 7 & Canadian occupational performance measure (COPM) & 1.3 \\
\hline 8 & SF-12 or SF-36 & 43.9 \\
\hline 9 & Others* & \\
\hline
\end{tabular}

Scale (UEFS) (90\%). See Table 8 for a full list of self-report measures reported.

When respondents were asked to provide the name of any other outcome measures, they gave a variety of answers. Other impairment measures include 9 hole peg test, Valpar 4, Purdue pegboard, pinch meter, and O'Connor tweezer test. While other self-report measures included ASES-elbow, Quick DASH, patient self-reporting of satisfaction and some non-standardized measures were occasionally specified.

\section{DISCUSSION}

This study surveyed upper extremity therapists, to determine current practice patterns and clinical beliefs pertaining to elbow fracture rehabilitation. Hand therapists with upper extremity certification formed the majority of the respondents. Expert opinion is considered the lowest level of evidence to support clinical practice; but it is acknowledged that its quality is enhanced by consensus exercises. Thus, this survey which identifies some areas of expert consensus can provide some support for elbow fracture management; and allows for examination of the gaps between evidence and practice.

Our results indicate that patient education is perceived as an essential component of elbow fracture rehabilitation throughout all phases of fracture healing. High levels of consensus on the use of AROM and AAROM exercises were achieved with respect to the acute stages, whereas functional performance was the focus beyond 6 weeks post injury. Unfortunately, the evidence to support elbow fracture rehabilitation is sparse [4]; although it does support early active motion. The use of ultrasound without concordance; which is not surprising given the lack of clear evidence.

Static splinting has been reported to be very effective in protecting the repaired structure and to facilitate healing [6]. It is of paramount importance in the immediate postoperative phase and the early joint protection phases of rehabilitation. Usually these splints are used anywhere between 2 weeks to 8 weeks post-operatively and this depends on the stability of the fracture and the severity of the injury [6]. However in the current survey only $58 \%$ of them 
have reported to use it regularly. Survey methods do not allow us to explore the reasons for responses. However, it may be possible that other methods of immobilization are used in the acute phase that were not specified.

A systematic review by Murdoch et al, described how weakness of the muscles surrounding the fracture site can persist long after full bone healing has occurred [7]. Similarly, it has been reported that return of strength may take 6 months or more following a fracture [8]. In our survey, only $4 \%$ of the respondents reported utilizing strengthening exercises regularly within the first 6 weeks of injury. There has always been a debate in the literature regarding the right timing to initiate strengthening exercises in rehabilitation after elbow fracture. However, before initiating a strengthening exercise protocol, important factors such as the type of fracture, the duration of fracture and the severity need to be taken into account. Muscle strengthening is typically avoided when it is thought it might compromise the stability of the fracture site. Progressive strengthening exercises can be initiated for the entire upper extremity after splint removal for simple uncomplicated fractures. Exercise can start with hand strengthening; and is followed by isometrics of the elbow and forearm as pain decreases. In more complex fractures strengthening may be started only after 8 to 12 weeks [9]. In hand centres the proportion of complex cases might be higher; and this is reflected in low rate of early strengthening exercises. The current data indicate that the early focus is on regaining joint range of motion.

In our study only $17 \%$ of the therapists reported "always or frequently"using ultrasound during the acute stage of healing. The effectiveness of pulsed ultrasound is proven in distal radius fracture [10]. Low-intensity, non-thermal, and pulsed were the parameters proven to accelerate the bone healing process $[10,11]$. There is no strong evidence to support the effectiveness of pulsed ultrasound in accelerating bone healing in elbow fractures. The distal radius fracture is a relatively less complicated area than the elbow fractures hence we cannot generalize the findings for distal radius fractures to elbow fractures. Further the dosage needed to promote bone healing is quite different than needed to treat pain and swelling; and we did not explore indications or dosage to see why ultrasound was used by some respondents. There appears to be a knowledge gap in this area that should be addressed in future research.

The most commonly reported exercise interventions for the treatment of elbow fracture at the functional rehabilitation stage are AROM, stretching, strengthening, functional exercise, PROM, and AAROM. This is consistent with the early focus on joint motion; and the fact that AROM is the predominant outcome measure. Bano et al. [12]. have proposed a protocol for elbow fracture rehabilitation which included all of the above interventions on which the therapists in our survey agreed upon.

Heat was the only modality that was reported to be used "always or frequently"by therapists, and was also reported to be the most effective as an adjunct in facilitating joint motion by increasing tissue extensibility [13]. A doubleblind repeated measures trial by Robertson et al. [14] reported that deep heating increases tissue extensibility in the absence of stretching, and that superficial heating is better than no heating at all. There are no specific studies that focus on the effect of heat modalities in elbow fracture rehabilitation. The fact that there is evidence supporting the use of heat to improve tissue extensibility suggests that further studies on defining and optimizing its usage for elbow fracture rehabilitation are warranted.

In the survey, massage was reported as a commonly used "hands-on" treatment. There is a lot of literature on the effects of massage, but researchers are not in complete agreement due to the variable quality of the available evidence. A recent systematic review reported that the trial evidence supported the effectiveness of massage for a range of conditions, but not specifically as a treatment for post elbow fracture [15]. Van den Dolder et al. [16] found that massage increased ROM and decreased pain in subjects with shoulder pain after 6 treatments. However, there are no specific studies addressing the effectiveness of massage in elbow fracture rehabilitation and it would be premature to make any strong recommendations from the results of this survey.

MWM was the second most commonly used manual therapy technique in the rehabilitation stage. The extent to which respondents reported using this technique to treat elbow fractures is interesting as it is not a component of entry-level education and learned through post-graduate clinical courses. This technique is a part of the Mulligan concept for mobilizations, and has been found to be effective in improving pain and restoring function post colles fracture when pain is the predominant limitation [17]. The role of MWM in elbow fracture rehabilitation is yet to be explored and no strong conclusions can be made based on the current literature base.

It is useful for therapists to agree upon core outcome measure to evaluate a specific patient population because it allows comparability across centers and studies on the outcomes achieved. The most commonly used outcome measures were goniometry, grip strength (Jamar), HHD, MMT, and the NPRS. These outcome measures are impairment-based rather than activity-based. This finding is consistent with previous studies showing similar practice patterns of distal radius fractures [18]. The focus on strength measures in initial assessment but not during treatment is an interesting discordance.

It is interesting to note that self-report outcome measures used in other areas of hand therapy practice as well as measures specifically designed for the elbow are not commonly used as outcome measures for elbow rehabilitation. The Disabilities of the Arm, Shoulder, and Hand (DASH) has been demonstrated to be a valid and reliable tool across a wide variety of upper extremity conditions including proximal and distal disorders of the upper extremity [19-21] The Patient-Rated Elbow Evaluation (PREE) measures pain and disability in patients with elbow disorders [22-24] and has been used in a variety of elbow populations. The Upper Extremity Functional Scale (UEFS) has been compared to the DASH and the Upper Limb Functional Index (ULFI) and was determined to be comparable in validity and reliability [25]. When asked if there was another measure that respondents use that was not an option in the survey, $19 \%$ (72.2\% of those who said "other") reported that they use the Purdue Pegboard Test. 
This test measures fine manual dexterity and was determined to be reliable and valid for those with a disability and limited functional capacity secondary to impairment of the upper limb, hand injury, Raynaud's disease, rheumatoid arthritis, and diabetes mellitus [26]. The validity of using a measure of fine dexterity in the assessment of elbow function is questionable; particularly given the fact that more relevant tests are available. For example, dexterity tests that would assess forearm rotation (i.e. The Minnesota Rate of Manipulation) or performance-based tests that assess gross motor function such as the FIT-HANSA [27] might be more appropriate. The FIT-HANSA is a recent publication, so it was not unexpected, that it would not be in common usage.

It was reported that a lesser number of hand therapists use self-report measures; this is a stark contrast to the high reliance on impairment measures. Since previous research has demonstrated that self-report measures are actually better predictors of return to work than are impairment measures [28], it would seem that this is a notable gap between evidence and practice.

Clinicians need to be aware of reported validity, reliability, internal consistency, responsiveness and minimal detectable change of the measures they are considering, as well as the patient population they were developed for and tested in to make a decision as to which is the most appropriate measure to use. It may be that the lack of use of self-report measures with respect to elbow rehabilitation is in part due to the lack of psychometric studies in this area. However, given that a similar trend was observed with respect to distal radius fractures [18] where numerous psychometric studies are available, this explanation alone does not suffice. Therapists who rehabilitate patients with an elbow fracture need to be in agreement on core outcome measures in order to evaluate change against a standard. With the move toward a hand therapy database, it would be an opportune time to improve the use of understanding of self-report measures, as these are more consistent with a functional rehabilitation objective.

Overall, linking evidence to practice indicates substantial gaps in knowledge. The development of evidence-based clinical practice guidelines should be based on strong evidence, clinical consensus and patient input. This survey could inform that process; but, in the absence of strong evidence differentiating different treatment options it would not be possible to define clear evidence-based guidelines at this time.

This study provides a sense of what constitutes a typical elbow fracture rehabilitation. However, the findings must be considered in light of study limitations. The number of certified hand therapists was 2340; but we do not know if the email was received by all; so we cannot determine a response rate. The access to Canadian and American Physical therapists was limited; thus our response primarily reflects the views of Occupational therapists who were hand therapists. Further, it was our intent to represent the views of therapists in both Canada and the United States, but only 8 of the respondents were from Canada. These findings suggest that the practice patterns may not be pertinent to therapists outside of the United States.
The structure of our survey may have affected the reporting. We divided management into acute and rehabilitation stages of fracture healing; but, therapists may not have been able to accurately answer within these classifications. Additionally, we may not have captured all possible treatment interventions within our survey options. Furthermore, respondents may have had difficulty assessing effectiveness; particularly for interventions they seldom used; or ones that they used for a specific indication. Finally, practice patterns were self-reported; and we do not know the reliability or validity of the reporting; nor were we able to establish cause and effect relationships.

Implications for Practice: This study described current practice in elbow fracture rehabilitation defining many areas of consensus including the early focus on regaining motion through the use of active exercise. However, there are large gaps between practice and supporting evidence including how to optimize regaining motion. Future research is now needed to determine the effectiveness of primary and adjunctive interventions, as well as the optimal treatment parameters including frequency, intensity, and duration of these interventions.

\section{CONCLUSION}

This study has identified general practice patterns currently used by hand therapists in North America. This survey has highlighted exercise, education, and functional activity as primary components of elbow fracture rehabilitation. Future research should focus on defining the optimal dosages, progressions and types of exercise/ activities, as well as core measures to monitor outcomes of these interventions. The findings of this study will help direct further studies in the area of elbow fracture rehabilitation, leading to the development of evidence-based CPGs to assist therapists in best practice for this population.

\section{CONFLICT OF INTEREST}

The authors confirm that this article content has no conflicts of interest.

\section{ACKNOWLEDGEMENTS}

Funded by a New Investigator Award, Canadian Institutes of Health Research.

\section{REFERENCES}

[1] Yian E, Karunakar M. Distal humerus fractures. American Academy of Orthopaedic Surgeons. 2007. Available from: http://orthoinfo.aaos.org [Accessed 5th Jan, 2012].

[2] Nance J, Lazaro R, Umphred D. The effect of early physical therapy intervention following elbow fracture on a postmenopausal female with multiple medical problems. J Phys Ther Sci 2004; 16(2): 159-64.

[3] Slocum Centre for Orthopedics and Sports Medicine. Elbow Problems. 2007. Available at http://www.slocumcenter.com [Accessed 25th Feb, 2012].

[4] MacDermid JC. What is the optimal rehabilitative approach to posttraumatic elbow stiffness. In: Wright T, Ed. Evidence-based orthopedics. Bethseda: Elsevier, Graphic World Publishing Services 2008.

[5] Roche N, Durieux P. Clinical practice guidelines: From methodological to practical issues. Intensive Care Med 1994; 20(8): 593-601.

[6] Wolff A. Elbow fractures and dislocations In: Cioppa-Mosca J, Cahill JB, Tucker CY, Eds. Postsurgical rehabilitation guidelines for the orthopedic clinician. St. Louis, Mo.: Mosby Elsevier 2006. 
[7] Murdoch A, Taylor N, Dodd K. Physical therapists should consider strength training as part of fracture rehabilitation. Phys Ther Rev 2004; 9(1): 51-9.

[8] Rocca GD. Olecranon fractures of the elbow. American Academy of Orthopaedic Surgeons. 2007. Available at http://orthoinfo.aaos. org [Accessed 5th Jan, 2012].

[9] Badia A, Stennett C. Sports-related injuries of the elbow. J Hand Ther 2006; 19(2): 206-26.

[10] Kristiansen TK, Ryaby JP, McCabe J, Frey JJ, Roe LR. Accelerated healing of distal radial fractures with the use of specific, low-intensity ultrasound. A multicenter, prospective, randomized, double-blind, placebo-controlled study. J Bone Joint Surg Am 1997; 79(7): 961-73.

[11] Busse JW, Bhandari M, Kulkarni AV, Tunks E. The effect of lowintensity pulsed ultrasound therapy on time to fracture healing: An analysis. CMAJ 2002; 166(4): 437-41.

[12] Bano KY, Kahlon RS. Radial head fractures - advanced techniques in surgical management and rehabilitation. J Hand Ther 2006; 19(2): 114-36.

[13] Knight C, Rutledge C, Cox M, Acosta M, Hall S. Effect of superficial heat, deep heat, and active exercise warm-up on the extensibility of the plantar flexors. Phys Ther 2001; 81(6): 120614.

[14] Robertson VJ, Ward AR, Jung P. The effect of heat on tissue extensibility: a comparison of deep and superficial heating. Arch Phys Med Rehabil 2005; 86(4): 819-25.

[15] Ernst E, Pittler MH, Wider B, Boddy K. Massage therapy: is its evidence-base getting stronger. Complement Health Pract Rev 2007; 12(3): 179-83.

[16] Van den Dolder PA, Roberts DL. A trial into the effectiveness of soft tissue massage in the treatment of shoulder pain. Aust $\mathrm{J}$ Physiother 2003; 49(3): 183-8.

[17] Naik VC, Chitra J, Khatri S. Effectiveness of Maitland versus Mulligan mobilization technique following post surgical management of colles fracture - RCT. Ind J Physiother Occup Ther 2007; 1(4): 14-8.
[18] Michlovitz SL, LaStayo PC, Alzner S, Watson E. Distal radius fractures: therapy practice patterns. J Hand Ther 2001; 14(4): 24957.

[19] Hudak PL, Amadio PC, Bombardier C. Development of an upper extremity outcome measure: the DASH (disabilities of the arm, shoulder and hand) [corrected]. The Upper Extremity Collaborative Group (UECG). Am J Ind Med 1996; 29(6): 602-8.

[20] Beaton D, Katz J, Fossel A, Wright J, Tarasuk V, Bombardier C. Measuring the whole or the parts? validity, reliability, and responsiveness of the DASH outcome measure in different regions of the upper extremity. J Hand Ther 2001; 14(2): 128-46.

[21] Westphal T, Piatek S, Schubert S, Schuschke T, Winckler S. Reliability and validity of the upper limb DASH questionnaire in patients with distal radius fractures. Z Orthop Ihre Grenzgeb 2002; 140(4): 447-51.

[22] John M, Angst F, Pap G, Junge A, Mannion AF. Cross-cultural adaptation, reliability and validity of the Patient Rated Elbow Evaluation (PREE) for German-speaking patients. Clin Exp Rheumatol 2007; 25(2): 195-205.

[23] MacDermid JC. Outcome evaluation in patients with elbow pathology: issues in instrument development and evaluation. J Hand Ther 2001; 14(2): 105-14.

[24] MacDermid JC, Michlovitz SL. Examination of the elbow: linking diagnosis, prognosis, and outcomes as a framework for maximizing therapy interventions. J Hand Ther 2006; 19(2): 82-97.

[25] Gabel PB, Urkett B. The upper limb functional index: development and determination of reliability, validity, and responsiveness. J Hand Ther 2006; 19(3): 328-48.

[26] Tiffin J, Asher EJ. The purdue pegboard: norms and studies of reliability and validity. J Appl Psychol 1948; 32(3): 234-47.

[27] MacDermid JC, Ghobrial M, Quirion KB, et al. Validation of a new test that assesses the functional performance of the upper extremity and neck (FIT-HaNSA) in patients with shoulder pathology. BMC Musculoskelet Disord 2007; 8: 42.

[28] MacDermid JC, Roth JH, McMurty R. Predictors of time lost from work following a distal radius fracture. J Occup Rehabil 2007; 17(1): 47-62. 